Interim Progress Report DE-FG07-96ER62305

\title{
A Fundamental Study of Laser-Induced Breakdown Spectroscopy Using Fiber Optics for Remote Measurements of Trace Metals
}

\section{Scott R Goode and S. Michael Angel}

The long-term goal of this project is to develop a system to measure the elemental composition of unprepared samples using laser-induced breakdown spectroscopy, LIBS, with a fiber-optic probe.

\section{Research Accomplishments (Year 1)}

- First Demonstration of LIBS Imaging Using Fiber Optics

- Combined LIBS and Raman Spectral Imaging in a Single Probe

- Established a Collaboration with David Cremers at LANL

- Developed a Method to Improve Launch Efficiency of High-Power Laser Pulses into an Optical Fiber Without Catastrophic Damage

- Applied AOTF to Plasma Imaging to Characterize the Plasma both Spatially and Temporally

- Development work near completion for high-resolution LIBS spectrometer

\section{Publications Resulting From or Related to this Work}

B.J. Marquardt, B.M. Cullum and S.M. Angel "Fiber-Optic Probe for Determining Heavy Metals in Solids Based on Laser-Induced Plasmas," in Proceedings, European Symposium on Environmental Sensing III, Munich, FRG, June 16-20, 1997, SPIE 310540 (1997).

Brian J. Marquardt, Scott R. Goode and S. Michael Angel, "In-Situ Determination of Lead in Paint by Laser-Induced Breakdown Spectroscopy Using a Fiber-Optic Probe," Anal. Chem., 68, 977-981 (1996).

Ph.D. Dissertation: Brian Marquardt, "Remote In-Situ Laser-Induced Breakdown Spectroscopy Using Optical Fibers." 11/7/97

\section{Conference Papers Resulting from this Work}

S. Michael Angel, H. Trey Skinner and Brian J. Marquardt, "Imaging Spectroscopy Using Optical Fibers," invited talk in the Anachem Award Symposium, 24th Annual Conference of the Federation of Analytical Chemistry and Spectroscopy Societies (FACSS), October 26-30, Providence, RI, Abstract 305 (1997). 
Brian Marquardt, Brian M. Cullum, Tim J. Shaw and S. Michael Angel, "Spatial and Temporal Profiling of Laser-Induced Plasmas," 24th Annual Conference of the Federation of Analytical Chemistry and Spectroscopy Societies (FACSS) October 26-30, Providence. RI. Abstract 353 (1997).

S.M. Angel, H.T. Skinner and B.J. Marquardt, "Imaging Spectroscopy Using Fiber-optic Probes," Microscopy and Microanalysis, Cleveland, Invited Paper 623, OH August, 1997.

Brian J. Marquardt, Scott R. Goode and S. Michael Angel, "Spatial Profiling of LaserInduced Plasmas for the Purpose of Optimizing Fiber-Optic LIBS Probe Designs," The Pittsburgh Conference on Analytical Chemistry and Applied Spectroscopy, Atlanta, Georgia, March 16-21, Abstract 816 (1997).

Brian J. Marquardt, Scott R. Goode, Timothy J. Shaw and S. M. Angel, "The Development of an In-Situ Method for Elemental Analysis Using Laser-Induced Breakdown Spectroscopy," 23rd Annual Conference of the Federation of Analytical Chemistry and Spectroscopy Societies (FACSS), Sept. 29-Oct. 4, Cincinnati, Ohio. Abstract 581 (1996).

Brian J. Marquardt, Scott R. Goode, Timothy J. Shaw and S. M. Angel, "The Development of a Method for Remote Elemental Analysis UsingLaser-Induced Breakdown Spectroscopy," 23rd Annual Conference of the Federation of Analytical Chemistry and Spectroscopy Societies (FACSS), Sept. 29-Oct. 4, Cincinnati, Ohio, POSTER Abstract 390 (1996).

\section{Patent Disclosure}

Brian J. Marquardt, Scott R. Goode, and S. Michael Angel. "On-Site Determination of Lead in Paint Using a Novel Optical Fiber Probe,” disclosed September 1996. 
Interim Progress Report DE-FG07-96ER62305

\section{Characterization of Laser-Induced Plasmas Using Temporal and Spatial Imaging Progress in Fundamental Measurements}

(Taken in part from the dissertation of Brian Marquardt, with permission. To be submitted for publication in Jan. 1998.)

\section{Introduction}

An experimental system was designed for spatially and temporally imaging laser-induced plasmas using coherent imaging bundles. The plasma images were spectrally resolved using an acousto-optic tunable filter (AOTF) and temporally resolved using an intensified charge coupled device (CCD). By spectrally imaging laser-induced plasmas, information about the stability and intensity of atomic emission can de determined and used to optimize fiber-optic LIBS collection.

The ability to achieve quantitative results using any analytical atomic emission technique requires that the method be calibrated. that the analysis configuration be optimized and that the analysis conditions be kept as uniform as possible. For LIBS, these parameters include the laser pulse characteristics, the sample characteristics (e.g. homogeneity, matrix composition, and moisture content) and the sampling geometry. The sampling geometry includes distance from the focusing lens to the sample, the method of focusing the laser to generate a plasma and the method for collecting the plasma emission. Recently, plasma imaging has been used to characterize laser-induced plasmas for optimizing sampling geometry. Multari and Cremers reported on the spatial and temporal distribution of $\mathrm{Cr}$ emission in a laser-induced plasma with respect to changes in the laser focusing lens-to-sample distance.' They concluded that the distance between the lens and the sample can greatly influence the analytical results of a LIBS experiment, Castle, et. al. captured time-resolved images of laser-induced plasmas created on a lead sample and report different emission profiles in the plasma images for ionic and atomic $\mathrm{Pb}$ emission. ${ }^{2}$ Other studies have also used time-resolved plasma imaging to determine the distribution of atomic and ionic species in the plasma plume. ${ }^{3-5}$ Bulatov et. al. reported two-dimensional spectral images of a LIBS plasma obtained by means of Fourier Transform visible spectroscopy to determine the distribution of chemical species in the plasma and reported that with their instrument they could obtain emission spectral at any point in the plasma for determining element distribution. ${ }^{6}$

Imaging can be a powerful tool for characterizing laser-induced plasmas for improving the sensitivity of LIBS measurements. Factors such as spatial plasma development and the distribution of atomic and ionic emission within the plasma over time need to be more thoroughly investigated to optimize the technique. By spatially and temporally imaging a plasma, information can be gained to improve the collection of atomic emission while reducing the effects of the continuum background. Using the information gained through plasma imaging fiber-optic LIBS probes can be designed to collect the atomic emission in the plasma at optimal locations leading to improved signal-to-noise in a LIBS measurement. These measurements may also lead to the development of optimized LIBS 
probes that would no longer require gated detection because the noise is reduced by spatial means instead of temporal.

Time-resolved spectral imaging of plasmas using an AOTF is a novel technique for providing spectrally filtered images of plasmas for determining element distributions. The use of an AOTF for spectral imaging has been previously described for Raman and fluorescence imaging applications. ${ }^{7.8}$ The AOTF, unlike a dispersing imaging spectrograph. allows for spatially resolved imaging of the entire laser-induced plasma.

The following report describes a method for obtaining spatially and temporally resolved images of plasmas using an acousto-optic tunable filter (AOTF) for spectral resolution of atomic $\mathrm{Pb}$ lines from a lead-containing solder sample. Spectral $\mathrm{Pb}$ images are presented showing the growth of plasmas both axially and orthogonally as a function of time. Plasma images also obtained remotely using a coherent imaging fiber are presented. A description of these images is given and data indicating the optimal regions for emission collection in these laser-induced plasmas is presented. A proposed probe design is presented that is optimized for collection of $\mathrm{Pb}$ emission in laser-induced plasmas for use with non-gated detection.

\section{Experimental}

The laser-plasmas were formed by focusing Q-switched Nd:YAG laser pulses (New Wave Research, Model Minilase 2-10, $1064 \mathrm{~nm}, 45 \mathrm{~mJ} /$ pulse) vertically onto a solder sample containing $60 \% \mathrm{~Pb}$. The plasma images were collected orthogonal to the incident laser beam using the system shown in Figure 1. The temporal and spatial plasma imaging system consisted of a $\mathrm{TeO}_{2}$-based acousto-optic tunable filter (AOTF) (Brimrose Corporation of America, Model TEAF7-.50-1.0-I), an intensified charge-coupled device (ICCD) detector (Princeton Instruments, Model ST-138 controller, Model ITEA/CCD576-S/RB-E detector) and collimating and focusing optics. The timing parameters for the temporally resolved imaging experiments were controlled using a programmable pulsedelay generator (Princeton Instruments, Model PG-200) to gate the ICCD. The AOTF was capable of continuous tuning of a narrow spectral bandpass $(3 \mathrm{~nm}$ at $650 \mathrm{~nm})$ from 500 to $900 \mathrm{~nm}$. For imaging the plasma orthogonally to the incident laser beam the light emitted by the plasma was collected and collimated using a f/4 (0.125 NA) plano-convex lens as shown in Figure 1. The collimated beam was passed through an aperture to minimize external light interference and then into the AOTF. The spectrally dispersed image was focused onto the ICCD detector using an f/6 (0.083 NA) plano-convex lens. The first-order diffracted beam from the AOTF was isolated from the zero-order beam by spatial filtering followed by a $32 \mathrm{~cm}$ dispersion path to the detector. The experimental design for collecting plasma images axially is similar to that used for orthogonal measurements and is shown in Figure 2. The timing parameters were the same for both the orthogonal and axial imaging experiments. The gate delays ranged from $50 \mathrm{~ns}$ to $3 \mu \mathrm{s}$ with images acquired every $25 \mathrm{~ns}$ and the gate width was set at a constant $250 \mathrm{~ns}$.

Plasma images were also obtained using a $350 \mu \mathrm{m}$ core diameter coherent fiber-optic imaging bundle (Sumitomo Electric, Model IGN-035/06) to collect and transfer the plasma image to the AOTF and ICCD as shown in Figure 3. The plasma was focused 
onto the face of the coherent imaging bundle using a 10x (0.25 NA) microscope objective. The image from the fiber was collimated with a f/2 (0.25 NA) plano-convex lens and spectrally dispersed using the AOTF and then focused onto the ICCD. The spectrally dispersed image was then focused onto the ICCD detector using an F/6 (.083 NA) plano-convex lens.

\section{Results and Discussion}

Spatially and temporally resolved orthogonal images of the laser-induced plasmas created on a $60 \% \mathrm{~Pb}$ solder sample were captured using the apparatus shown in Figure 1. The AOTF was tuned to one of three wavelengths for obtaining spatially resolved images. The $\mathrm{Pb}$ emission was imaged at $722.8 \mathrm{~nm}$, and the continuum background emission was measure at two separate wavelengths, $715.3 \mathrm{~nm}$ and $730.6 \mathrm{~nm}$. The images obtained both above and below the $\mathrm{Pb}$ emission wavelength were used to calculate the amount of noise due to continuum background emission at each delay time in the plasma. The average of the two background images was calculated and then subtracted from the $\mathrm{Pb}$ emission images at each delay time to accurately represent the distribution of $\mathrm{Pb}$ emission in the plasma. The plasma images were temporally resolved from $50 \mathrm{~ns}$ to $3 \mu$ s after the plasma was formed. Plasma images at all three wavelengths were collected every $25 \mathrm{~ns}$ over this range. Each image is the collected signal produced by one laser shot.

Figure 4 shows two sequences of plasma images at various delay times after the plasma was formed. The background subtracted images in Figure 4a show the growth and development of the $\mathrm{Pb}$ emission in the plasma over a $2.5 \mu$ s range of delay times. Initially, the $\mathrm{Pb}$ emission is localized at the outer edges of the expanding plasma with a small region beginning to rise above the surface at $50 \mathrm{~ns}$. The unusual appearance of the image at a delay of $50 \mathrm{~ns}$ is due to the subtraction of the intense continuum emission found at shorter delay times (Figure $4 b$ ). As the plasma grows in the subsequent images the most intense emission region shifts from the central regions in the plasma at early times to the upper regions of the rising plasma plume. The images in Figure $4 \mathrm{~b}$ show the corresponding continuum background images collected at $715.3 \mathrm{~nm}$ at the same delay times. As seen in Figure 4b, the background emission is very intense early in the plasma, but as the plasma begins to expand and cool the continuum emission quickly dissipates and is no longer evident at delay times greater than $1.9 \mu \mathrm{s}$ in this experiment. The height of the plasma after a delay of $2.5 \mu$ after plasma formation was calculated from the image of the plasma to be approximately $3.6 \mathrm{~mm}$. By looking at the spatial distribution of the plasma as a function of delay time in Figure 4a, it is apparent that the optimal region for collection in the plasma is very dependent on the delay time. It would be difficult to perform analytically precise LIBS measurements from signal collected orthogonally to the incident laser without first performing a detailed experiment to determine the optimal delay time for the sample of interest. It should also be noted that at longer delay times the plasma images in Figure 4 seems to shift spatially from the center as delay times increase. These types of spatial shifts in the plasma can easily lead to increased noise and a decrease in the overall precision of a LIBS measurement. The reason for the shift in the plasma plume was not investigated. 
Many of our current fiber-optic LIBS probes are designed to collect the emission from the plasma axially along the same path as the incident laser beam. Therefore, in this study we were also interested in monitoring the growth of the plasma axially as a function of delay time to determine the optimal parameters for emission collection in our fiber-optic LIBS probes. Temporally and spatially resolved plasma images collected axially along the path of the incident laser are shown in Figure 5. The axial plasma imaging experiment was performed with the same timing parameters as the orthogonal imaging experiment. The delay times ranged from $50 \mathrm{~ns}$ to $3.0 \mu \mathrm{s}$ with images collected every $25 \mathrm{~ns}$ and a constant gate width of $250 \mathrm{~ns}$. The AOTF was set to collect the $\mathrm{Pb}$ emission at $722.8 \mathrm{~nm}$ and the background continuum images were obtained at 715.3 and $730.6 \mathrm{~nm}$. The background subtracted $\mathrm{Pb}$ emission images at $722.8 \mathrm{~nm}$ in Figure $5 \mathrm{a}$ show a general radial expansion of the plasma plume as the delay time increases. The unusual appearance of the plasma image in the first frame of Figure 5a is due to the subtraction of the very intense continuum background found early on in the plasma. The intensity of the background continuum as shown in Figure $5 \mathrm{~b}$ is significantly reduced in the axial plasma images compared to the orthogonal data shown in Figure $4 \mathrm{~b}$. The background images at later time delays appear different in Figure $5 \mathrm{~b}$ because they were normalized to the image at $675 \mathrm{~ns}$ for clarity, due to the weak intensity of the signal The images were normalized by factors of 3,4 and 7 for the images at delay times of 1.3, 1.9 and $2.5 \mu \mathrm{s}$, respectively. In terms of optimizing the collection efficiency of a LIBS measurement, the axial plasma images expand radially approximately $30 \%$ less than the plume expands vertically in the orthogonal measurements in the same amount of time. The axial plasma plume at a delay time of $2.5 \mu \mathrm{s}$ has a diameter of $2.6 \mathrm{~mm}$ and the height of the plasma from the sample was $3.5 \mathrm{~mm}$ at the same delay time. The spatial shift mentioned for the later delay times for the orthogonal measurements is also apparent in the axial images at later delay times.

Orthogonal plasma images were also obtained remotely using a $350 \mu \mathrm{m}$ core diameter coherent imaging optical fiber. The plasma was imaged onto the proximal end of the $1 \mathrm{~m}$ long imaging fiber using a 10x microscope objective, the image was then collimated using a f/2 plano-convex lens, The appropriate wavelength was selected using the AOTF and focused onto the ICCD chip. The experiment was performed for gate delays between $100 \mathrm{~ns}$ and $3000 \mathrm{~ns}$ collecting an image every $100 \mathrm{~ns}$. The gate width was $200 \mathrm{~ns}$ for the fiber-optic plasma imaging experiment. The signal obtained at each delay time was an accumulation of 20 laser pulses. The signal from a number of laser shots had to be accumulated because of losses incurred using the small diameter imaging fiber. Plasma images of individual laser pulses should be possible with larger diameter imaging fibers, Two sequences of plasma images formed on the same $\mathrm{Pb}$ sample are shown in Figure 6. The sequence of images in Figure 6a were obtained with the AOTF set at $722.8 \mathrm{~nm}$ to show the spatial and temporal growth of the $\mathrm{Pb}$ emission in the plasma. The images in Figure 6a have had the average continuum background from images taken at $730.6 \mathrm{~nm}$ and $715.3 \mathrm{~nm}$ subtracted from them. The unusual appearance of the first image in that sequence at $100 \mathrm{~ns}$ is due to the subtraction of the very intense continuum emission at that early delay time. The temporal $\mathrm{Pb}$ emission profiles in Figure 6a agree very well with the emission profiles shown in figure 4a. At later delay times $(>2 \mu \mathrm{s})$ the plasma has actually expanded vertically out of the collection area of the 10x microscope 
objective. This is a problem mainly due to the small diameter of the imaging fiber. The entire plasma image could easily be collected using a larger diameter imaging fiber. The images in Figure $6 \mathrm{~b}$ show the emission from the continuum background collected at $715.3 \mathrm{~nm}$ The size of the full image area of the imaging fiber can be seen in the continuum image at $100 \mathrm{~ns}$ in Figure 6b. Like the images in Figure 4b there is very little continuum emission collected beyond time delays of $1.5 \mu$ s. The use of imaging optical fibers for the collection of plasma images allows great flexibility in developing experiments to characterize the temporal and spatial characteristics of a laser-induced plasma. For example, any number of imaging fibers with attached imaging GRIN lenses could be positioned to image a plasma at various positions simultaneously. This would allow for detailed characterization of each laser pulse and can lead to improvements in the LIBS collection system to improve both the precision and sensitivity of the measurement.

To design a fiber-optic LIBS probe optimized for the collection of $\mathrm{Pb}$ emission axially in a laser-induced plasma two factors were considered. First, what region of the plasma has the highest intensity emission signal and the secondly, what region of the plasma has significant emission signal and little contribution due to background noise. By finding a region with significant emission and little background it is possible to obtain quality LIBS spectra without a gated detector, therefore making a LIBS instrument far less expensive. To determine an optimal region for performing these experiments the main consideration was maximizing the $\mathrm{Pb}$ emission signal and minimizing the background. The image in Figure 7 is of a Pb plasma obtained at a delay time of $2.5 \mu \mathrm{s}$, the region of interest (ROI) in the outer region of the plasma image indicates the area used for measuring the signal and noise intensity for the different delay times. The mean intensity value for the ROI was obtained for each non-background subtracted $\mathrm{Pb}$ image and for each background image. The ratio of the mean intensity values for both the $\mathrm{Pb}$ signal and the background signal were plotted versus delay time as shown in Figure 8. For that region in the plasma the signal-to-noise ratio was approximately 3 times higher at $2.5 \mu \mathrm{s}$ than at $100 \mathrm{~ns}$. With this difference in the signal-to-noise ratio it may be possible to perform non-gated LIBS experiments if the collection system was focused at this region of interest. Determining the optimal vertical position to collect from above the sample could also improve the signal-to-noise ratio for non-gated measurements.

To determine the optimal region for collecting emission signal in a $\mathrm{Pb}$ plasma with gated detection we calculated the ratio of the non background subtracted $\mathrm{Pb}$ emission images in Figure $5 \mathrm{a}$ to the respective background image in figure $5 \mathrm{~b}$. The plasma images at delay times of $100 \mathrm{~ns}$ and higher were summed together to create a single image shown in Figure 9 that is representative of the accumulated emission signal over that time period. From this image it is apparent that the more central region in the axial plasma contains the highest intensity emission signal. The contour plot in Figure 10 further illustrates the more intense emission in the central area of the axial plasma. The inner regions of the plasma because of the higher emission intensity would allow for the determination of lower detection limits using a LIBS system with gated detection to overcome the higher background in this region. 
Interim Progress Report DE-FG07-96ER62305

\section{Conclusions}

From images shown in this report it is evident that the temporal and spatial behavior of laser-induced plasmas Is a complex process. However, through the use of spectral imaging, optimal conditions can be determined for collecting the atomic emission signal in these plasmas. By tailoring signal collection to the regions of the plasma that contain the highest emission signal with the least amount of background interference both the detection limits and the precision of LIBS measurements could be improved. The optimal regions for both gated and possibly non-gated LIBS measurements have been shown to correspond to the inner regions and outer regions, respectively, in an axial plasma. By using this data fiber-optic LIBS probe designs can be optimized for collecting plasma emission at the optimal regions for improved detection limits and precision in a LIBS measurement.

\section{References}

1. R. A. Multari, L. E. Foster, D. A. Cremers and M. J. Ferris, Appl. Spectrosc., 50, 1483, (1996)

2. B. C. Castle. K. Visser. B. W. Smith and J. D. Winefordner, Appl. Spectrosc., 51, 1017: (1997).

3. J. M. Vadillo, M. Milan and J. J. Laserna, Fresenius J. Anal. Chem., 355, 10, (1996).

4. D. Romero and J. J. Laserna, Anal. Chem., 69, 2871, (1997).

5. D. B. Geohegan. Appl. Phys. Lett., 60, 2732, (1992).

6. V. Bulatov. L. Xu and I. Schecter, Anal. Chem., 68, 2966, (1996)

7. P. J. Treado, I. W. Levin and E. L. Lewis. Appl. Spectrosc., 46, 1211, (1992).

8. H. T. Skinner. T. F. Cooney and S. M. Angel, Appl. Spectrosc., 50, 1007, (1996) 


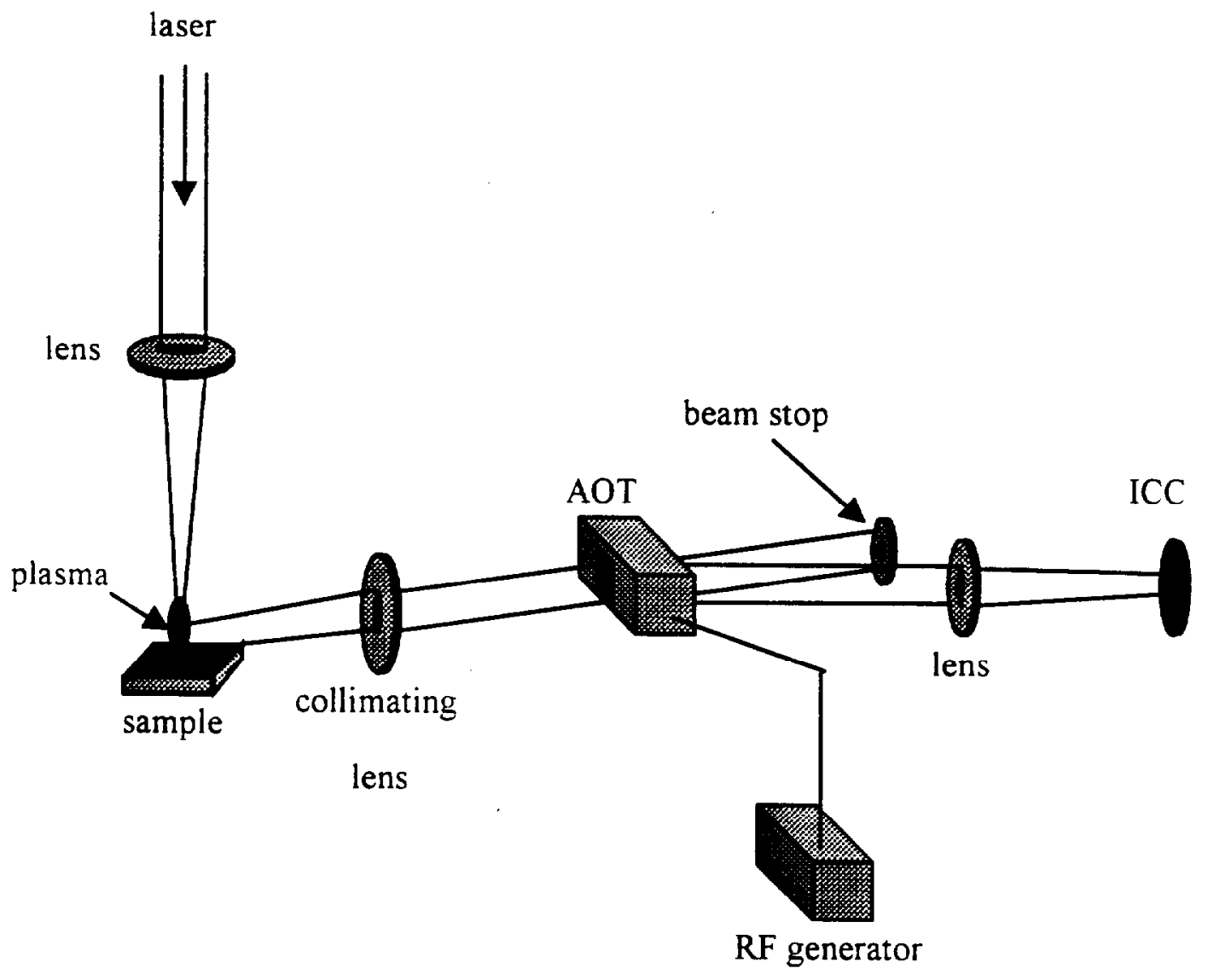

Figure 1 Experimental setup for obtaining time-resolved plasma images orthogonal to the incoming laser beam with conventional optics. 


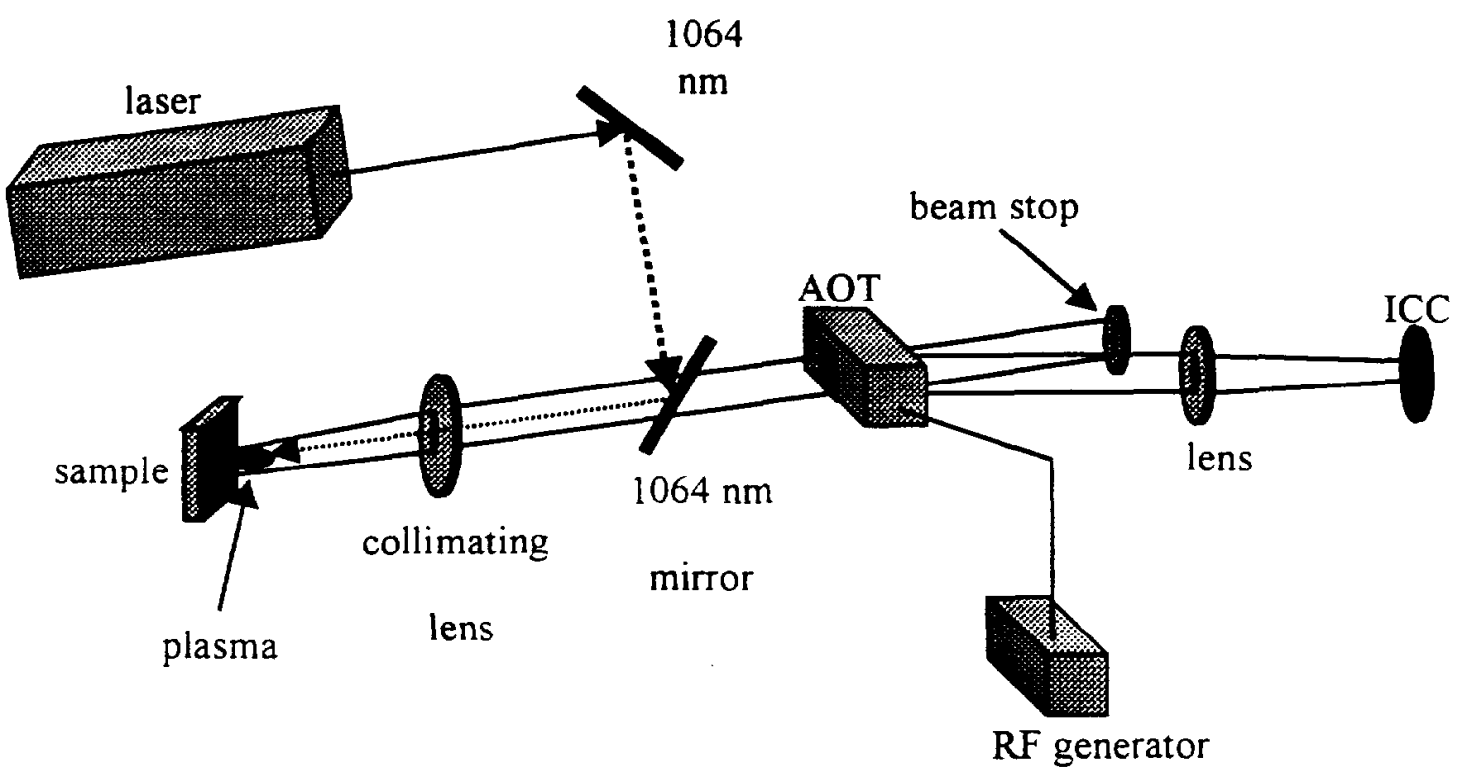

Figure 2 Experimental setup for obtaining time-resolved images along the same axis as the laser. 


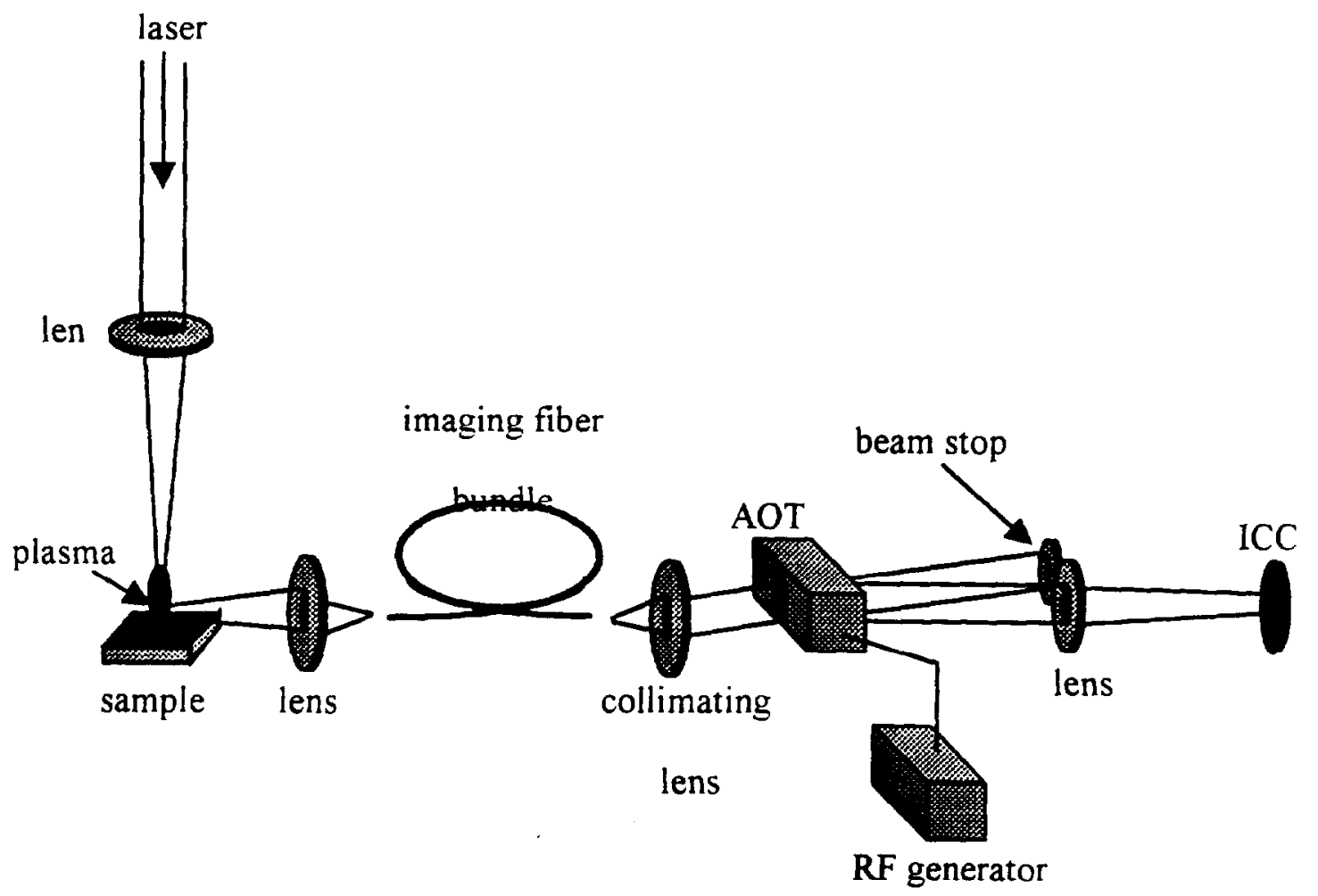

Figure 3 Same apparatus as in Figure 1 except that a coherent imaging fiber is used to collect and transfer the plasma images to the AOTF and detector. 


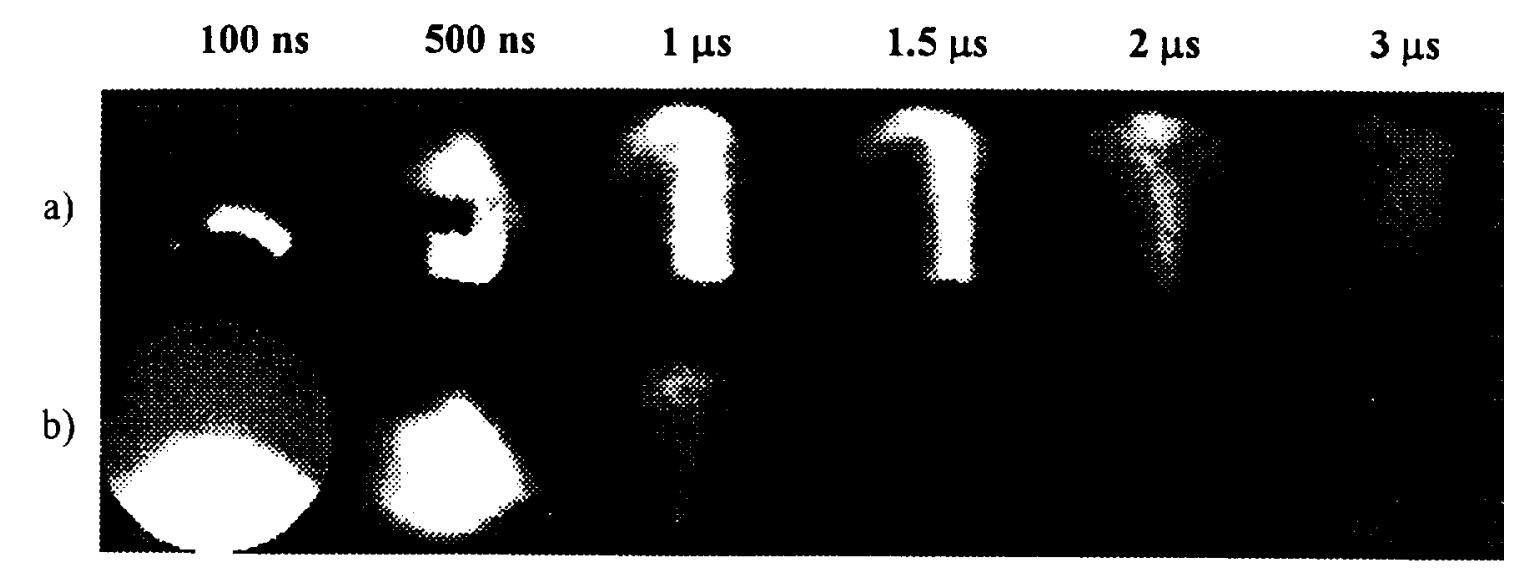

Figure 5 Plasma images collected axially (along the path of the laser) showing the time evolution of $\mathrm{Pb}$ emission (a) at $722.8 \mathrm{~nm}$ and continuum background emission (b) at $715.3 \mathrm{~nm}$ The images in sequence (a) were background subtracted to remove background emission due to the continuum. 


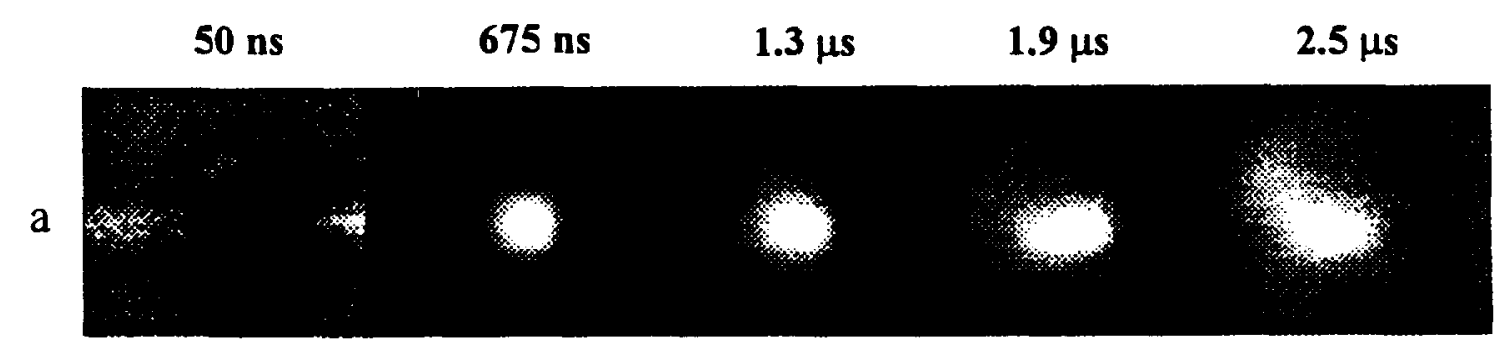

b

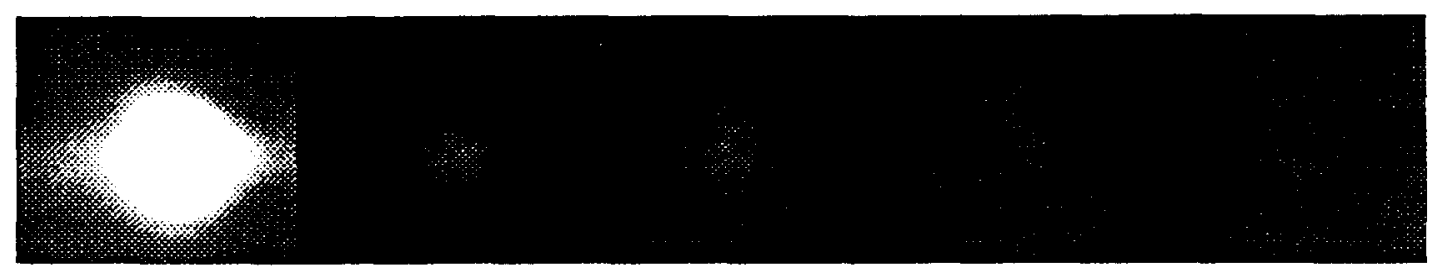

Figure 6 Plasma images collected orthogonal to the plasma axis using a $350 \mu \mathrm{m}$ coherent imaging fiber as a function of delay time. The $\mathrm{Pb}$ emission images in sequence (a) at $722.8 \mathrm{~nm}$ were background subtracted to remove the continuum emission shown in sequence (b) at $715 \mathrm{~nm}$. 
a)

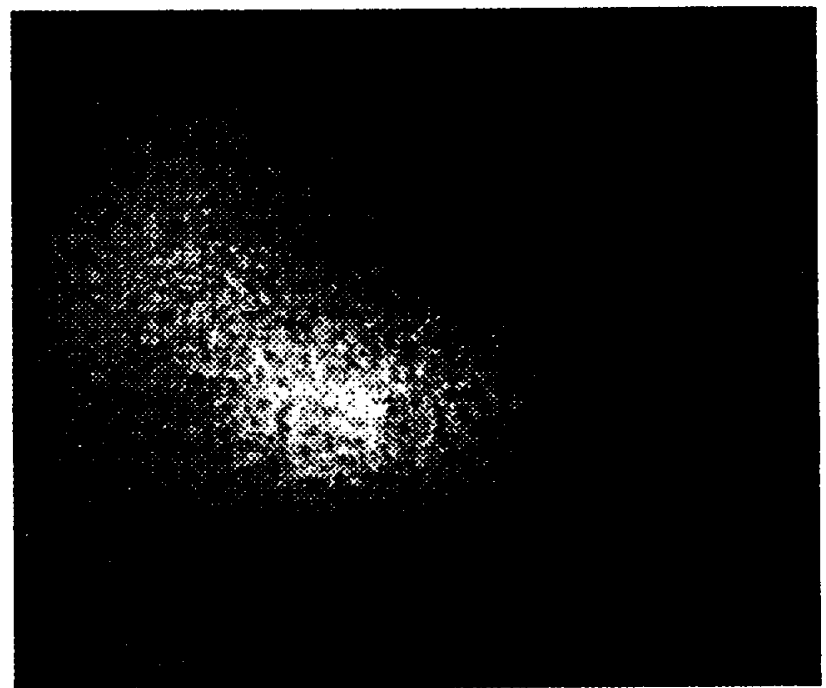

b)

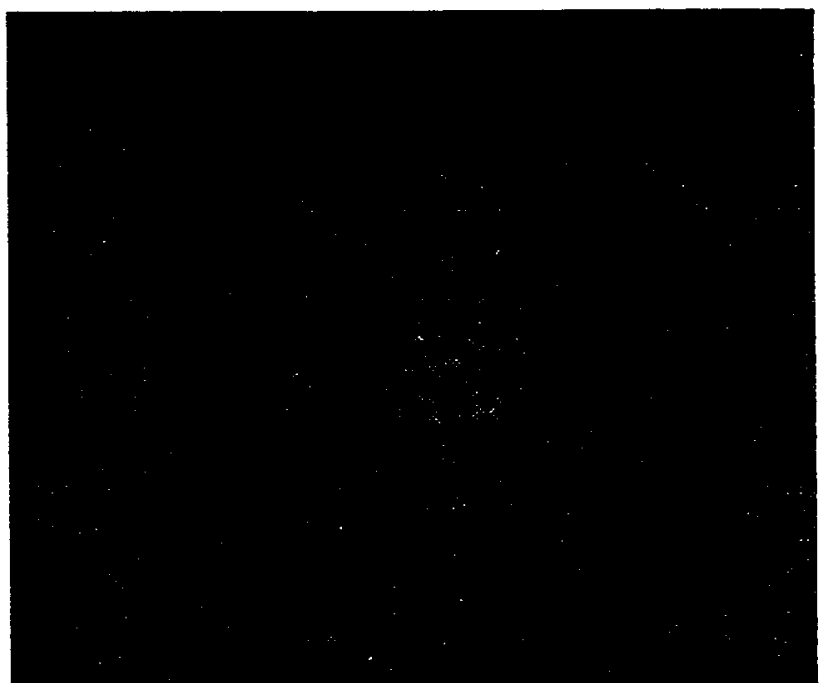

Figure $7 \quad$ Axial plasma image of $\mathrm{Pb}$ emission (a) collected at $722.8 \mathrm{~nm}$ and continuum background image (b) collected $715.3 \mathrm{~nm}$ at a delay time of $2.75 \mu \mathrm{s}$. The box indicates the region of interest (ROI) used to determine the possibility of spatially resolving emission collection to perform nongated LIBS measurements. 


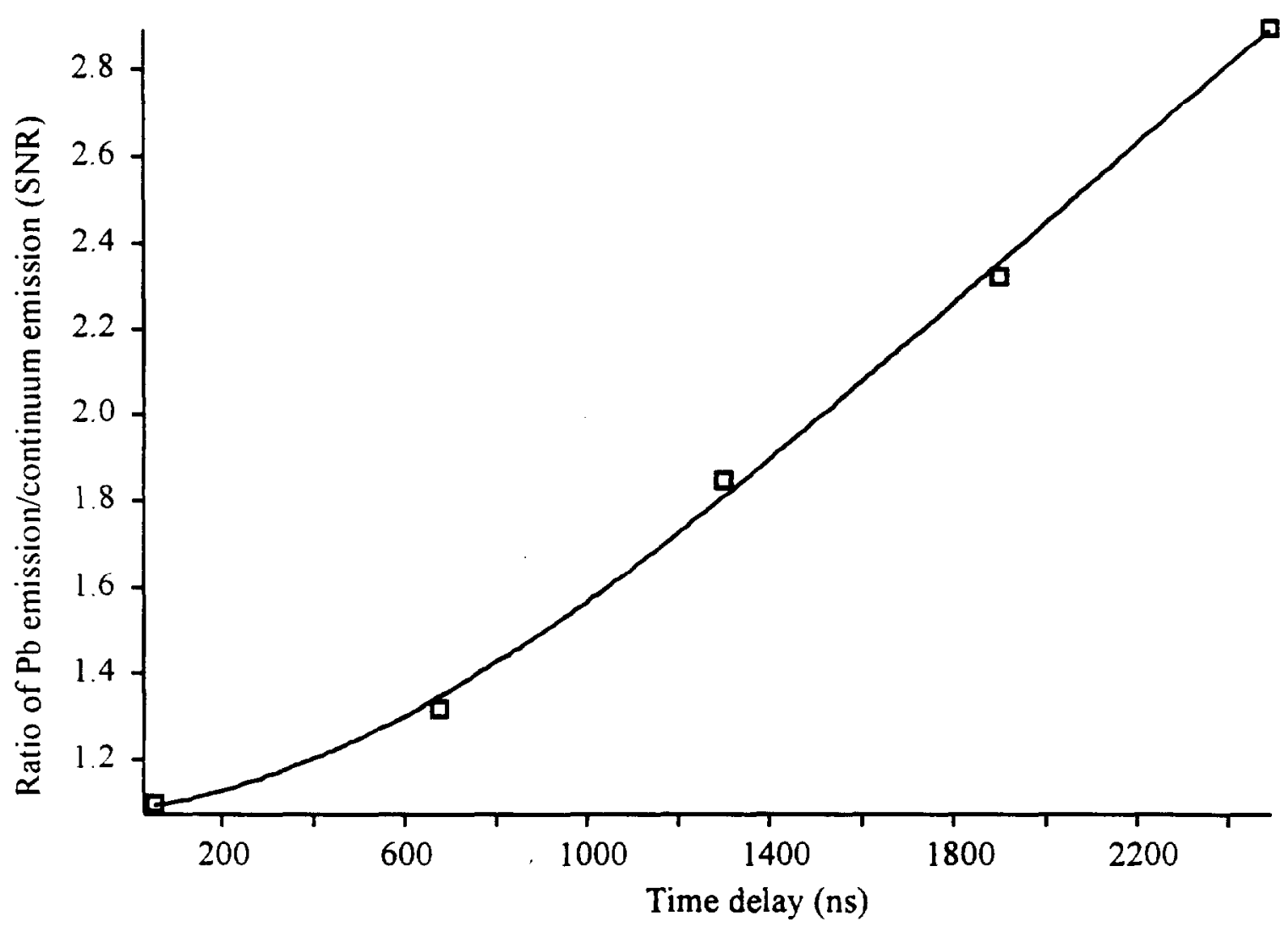

Figure 8 A plot of the ratio of the mean values calculated in the (ROI) shown in Figure 7 versus delay times ranging from 100 to $2.5 \mu \mathrm{s}$. 


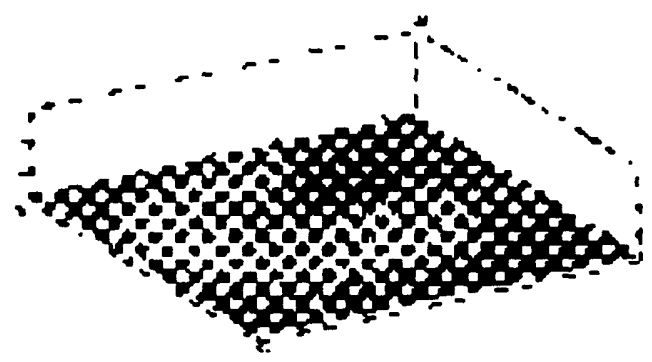

Figure 10 Perspective plot of Figure 9 more clearly illustrating the region for maximum signal collection using gated detection. 